\title{
Corrected local-density approximation band structures, linear-response dielectric functions, and quasiparticle lifetimes in noble metals
}

\author{
V. P. Zhukov, ${ }^{1}$ F. Aryasetiawan, ${ }^{2}$ E. V. Chulkov, ${ }^{1,3}$ I. G. de Gurtubay,${ }^{4}$ and P. M. Echenique ${ }^{1,3}$ \\ ${ }^{1}$ Donostia International Physics Center (DIPC), P Manuel de Lardizabal, 4, 20018, Donostia-San Sebastián, Spain \\ ${ }^{2}$ Joint Research Center for Atom Technology, Angström Technology Partnership, 1-1-4 Higashi, Tsukuba, Ibaraki 305, Japan \\ ${ }^{3}$ Departamento de Física de Materiales and Centro Mixto CSIC-UPV/EHU, Facultad de Ciencias Químicas, Universidad del Pais Vasco/ \\ Euskal Herriko Unibertsitatea, Apartado 1072, 20018 San Sebastián/Donostia, Basque Country, Spain \\ ${ }^{4}$ Materia Kondentsatuaren Fisika Saila, Zientzi Fakultatea, Euskal Herriko Unibersitatea, 644 Postakutxatila, \\ 48080 Bilbo, Basque Country, Spain
}

(Received 26 February 2001; revised manuscript received 6 June 2001; published 22 October 2001)

\begin{abstract}
We report the calculations of dielectric functions and quasiparticle lifetimes for noble metals $\mathrm{Cu}, \mathrm{Ag}$, and $\mathrm{Au}$ performed within the LMTO-GW approach. We show that by changing the value of continuous principal quantum number of $d$ states it is possible to improve the calculated local density approximation (LDA) band structures and dielectric functions. In particular, the plasmon absorption in $\mathrm{Ag}$, which is absent in the selfconsistent LDA approach, is obtained with the corrected band structure. We also show that the drawbacks of the LDA band structures do not lead to qualitative changes of the quasiparticle lifetimes. The lifetimes of electron excitations calculated by the LMTO-GW method agree well with the results of the LDA plane-wave $\mathrm{GW}$ approach. By adjusting the value of the density parameter $r_{s}$ good agreement with the results of the many-body free-electron gas calculations is also achieved for the electrons and holes in $s, p$-bands. For the holes in the top $d$ bands we report the lifetime band structures.
\end{abstract}

DOI: 10.1103/PhysRevB.64.195122

PACS number(s): 71.10.Ca, 71.10.Li, 71.15.Ap, 71.15.Mb

\section{INTRODUCTION}

The field of electron dynamics in metals has undergone extensive development during the last decades. The importance of such investigations is determined by the role that the hot electrons play in a variety of physical and chemical phenomena, including, e.g., chemical reactions on surfaces ${ }^{1}$ or electron correlation phenomena in the spectra of molecules and solids. ${ }^{2}$ A number of experimental techniques to study electron dynamics in solids have been developed, a very powerful being time-resolved two-photon photoemission spectroscopy ${ }^{3}$ (TR-2PPE) that allows the measurement of the hot electron lifetimes directly in a time domain. Using this kind of spectroscopy, a series of measurements of excited electrons lifetimes has been performed for nonmagnetic metals, ${ }^{4-11}$ ferromagnetic transition metals, ${ }^{12,13}$ and high- $T_{c}$ superconductors. ${ }^{14}$

The first theoretical evaluations of the hot electron lifetimes within the self-energy formalism of many-body theory were performed by Quinn and Ferrell ${ }^{15}$ for a free electron gas (FEG) model. Later a number of improved FEG calculations have been carried out based on the random-phase approximation $^{16}$ (RPA) and including also exchangecorrelation effects. ${ }^{17}$ In subsequent FEG-based researches statistical approximations were used ${ }^{18}$ and the inclusion of band-structure effects was discussed. ${ }^{19}$

Recently first principles calculations of the electron lifetimes in bulk noble metals $\mathrm{Cu}, \mathrm{Ag}$, and $\mathrm{Au}$ have also been published in Refs. 21-23,25 and 26. These evaluations employed a non-self-consistent GW approach that has proven its effectiveness in many previous researches. ${ }^{27,28}$ In the first step, the $a b$ initio $\mathrm{GW}$ calculations use the local density functional theory to evaluate the band structure of solids. Then the practical calculation schemes employ the linear response theory ${ }^{29,30}$ to compute the dynamical density response function of a solid. Based on RPA, the response function is used to compute the dielectric functions and screened Coulomb potential. Finally the matrix elements of the self-energy operator are calculated and the Dyson equation is solved to obtain the self-energy corrections to the local density approximation (LDA) eigenvalues. The quasiparticle characteristics obtained in this way, i.e., energies and lifetimes of excitations, usually show semiquantitative agreement with experimental data. However, many problems related to the approximations employed still remain unresolved.

One such problem is associated with the usage of LDA band structures in many-body calculations. Although the GW calculations can be performed self-consistently, non-selfconsistent computations appear to be more practical because they often produce excitation energies in better agreement with experimental data than the self-consistent calculations. $^{21,27,28}$ The success of the non-self-consistent $\mathrm{GW}$ approach is, however, dependent on the quality of the one-particle LDA eigenstates. Being invented to study the electronic ground state, the LDA approach is in principle not well suited to describing the excited states. So the direct usage of LDA to analyze excitations sometimes leads to essential disagreement with experiment. Hence, some wellknown problems appear, e.g., the "band-gap problem" in semiconductors. When used in the first step of many-body calculations, the LDA approach may also lead to some drawbacks. An example is the calculations of Ref. 24 for the dielectric functions of $\mathrm{Cu}$ and $\mathrm{Ag}$. While generally successful, these calculations failed to reproduce the most prominent part of the electron-energy-loss spectrum (EELS) of Ag, 
which is the peak of plasmon absorption with an energy about $3.8 \mathrm{eV}$. The absence of the plasmon peak is evidence of some drawbacks in the calculations for dielectric functions. Hence, the precision of lifetime calculations can be affected.

Some important problems are also related to the details of GW calculations. Almost all of the $a b$ initio evaluations of the quasiparticle lifetimes performed to date employed the plane-wave basis sets. ${ }^{21-23,25,26}$ Such computations are very time-consuming and hence several additional approximations are used. The size of dielectric function matrices is usually reduced to some tens of plane waves. The validity of using such small basis sets in the studies of excitations from the partly localized $d$ states of noble metals has still to be checked. The verification of the calculated lifetimes by comparing them with experimental data is, however, often problematic. One of the reasons is that different experimental researches for all the same objects under study often result in very divergent values of quasiparticle lifetimes (see, for example, the discussion of experimental data in Ref. 22).

A further reason why the results of the lifetime calculations deserve new verification is that in the cited papers very different methods were used to solve the Dyson equation. This may be the origin of some disagreements in the calculated lifetimes. In Refs. 22, 25 and 26 the so-called energy shell approximation with the renormalization factor equal to unity was employed to obtain the imaginary part of the quasiparticle energies, while in Refs. 21 and 23 direct search for Green's function poles was invoked. Such circumstances stimulate attempts to perform new improved lifetime calculations with different basis sets. In this paper we present the results of the calculations for dielectric functions and quasiparticle lifetimes in noble metals $\mathrm{Cu}, \mathrm{Ag}$, and $\mathrm{Au}$ by the linear muffin-tin orbital (LMTO)-GW method. In particular, we avoid using the energy shell approximation, performing precise calculations for the renormalization factor. The main advantage of the LMTO approach is that instead of large sets of plane waves it uses small sets of linear muffintin orbitals constructed from the numerical solutions of the Kohn-Sham equations inside atomic spheres. The numerical difficulties to calculate partly localized $d$ bands appear to be moderate here. One further favorable feature of this approach is that the basis sets for the polarization and dielectric functions are created using the products of the muffin-tin orbitals, so rather small basis sets appear to suffice.

Within the LMTO approach we propose a simple procedure to modify band states by changing the continuous principal quantum number of basis orbitals. Using this procedure we assess drawbacks in the calculated dielectric functions and quasiparticle lifetimes. Employing the advantage of small numerical basis sets we are able to distinguish within the multitude of data on lifetimes some branches that can be referred to for the symmetry directions in the Brillouin zone. For these directions we plot the dispersion laws of lifetimes for the first time. Finally, we discuss the applicability of the free-electron-gas theory to the calculations of lifetimes of free-electron-like quasiparticles.

\section{METHOD OF CALCULATIONS}

Within many-body perturbation theory ${ }^{29}$ the excited electrons are described by the dynamical equation for the Green function

$$
\begin{aligned}
& {\left[\omega-H_{0}(\mathbf{r})\right] G\left(\mathbf{r}, \mathbf{r}^{\prime}, \omega\right)-\int d^{3} \mathbf{r}^{\prime \prime} \Sigma\left(\mathbf{r}, \mathbf{r}^{\prime \prime}, \omega\right) G\left(\mathbf{r}^{\prime \prime}, \mathbf{r}^{\prime}, \omega\right)} \\
& \quad=\delta\left(\mathbf{r}-\mathbf{r}^{\prime}\right),
\end{aligned}
$$

where $H_{0}$ is the sum of the Hartree potential and kinetic energy and $\Sigma$ is the electron self-energy. The quasiparticle equation is defined by

$$
\begin{aligned}
& H_{0}(\mathbf{r}) \psi_{\mathbf{q}, i}(\mathbf{r}, \omega)+\int d^{3} \mathbf{r}^{\prime} \Sigma\left(\mathbf{r}, \mathbf{r}^{\prime}, \omega\right) \psi_{\mathbf{q}, i}\left(\mathbf{r}^{\prime}, \omega\right) \\
& =E_{\mathbf{q}, i}(\omega) \psi_{\mathbf{q}, i}(\mathbf{r}, \omega) .
\end{aligned}
$$

From the first-order perturbation theory we have the Dyson equation for the complex quasiparticle energy $E_{\mathbf{q}, i}(\omega)$,

$$
E_{\mathbf{q}, i}(\omega)=e_{\mathbf{q}, i}+\left\langle\psi_{\mathbf{q}, i}|\Delta \Sigma(\omega)| \psi_{\mathbf{q}, i}\right\rangle .
$$

Here $\quad \Delta \Sigma(\omega)=\Sigma(\omega)-V_{L D A}^{x c}, \quad V_{L D A}^{x c} \quad$ being the LDA exchange-correlation potential, so the matrix elements of Eq. (3), hereafter labeled $\Delta \Sigma_{\mathbf{q}, i}(\omega)$, are many-body self-energy corrections to the LDA eigenvalues $e_{\mathbf{q}, i}$. In practical $a b$ initio calculations the self-energy corrections are often calculated at the energy $\omega=e_{\mathbf{q}, i}$ : the so-called energy shell approximation, Refs. 22, 25 and 26 . Another way to simplify the solution of Dyson equation appears when only the linear part of the dependence of $\operatorname{Re} \Delta \Sigma$ on $\omega$ is retained and the change of $\operatorname{Im} \Delta \Sigma$ with $\omega$ is neglected, Refs. 27 and 28. In this approximation the self-energy corrections are

$$
\Delta e_{\mathbf{q}, i}=E_{\mathbf{q}, i}-e_{\mathbf{q}, i}=Z_{\mathbf{q}, i} \Delta \Sigma_{\mathbf{q}, i}\left(e_{\mathbf{q}, i}\right),
$$

where

$$
Z_{\mathbf{q}, i}=\left[1-\frac{\partial \operatorname{Re} \Delta \Sigma_{\mathbf{q}, i}(\omega)}{\partial \omega}\right]_{\omega=e_{\mathbf{q}, i}}^{-1}
$$

is the so-called renormalization factor. For the free-electron gas model this is a slowly varying function that is approximately equal to $0.8{ }^{30}$ Taking $Z_{\mathbf{q}, i}=1$ one has the energy shell approximation. In this paper we deal only with the imaginary part of the self-energy correction that gives the linewidth of the quasiparticle excitation and whose inverse determines the lifetime of a quasiparticle, ${ }^{27}$

$$
\tau_{\mathbf{q}, i}^{-1}=2 \times\left|\operatorname{Im} \Delta e_{\mathbf{q}, i}\right|
$$

We calculate the self-energy with the GW approximation of many-body theory, ${ }^{27,28}$ by retaining the first member in the series expansion of $\Sigma$ in terms of the screened Coulomb interaction $W$ :

$$
\Sigma\left(\mathbf{r}, \mathbf{r}^{\prime}, \omega\right)=\frac{i}{2 \pi} \int d \omega^{\prime} G\left(\mathbf{r}, \mathbf{r}^{\prime}, \omega+\omega^{\prime}\right) W\left(\mathbf{r}, \mathbf{r}^{\prime}, \omega^{\prime}\right) .
$$

The method of evaluating the matrix elements of the selfenergy operator within the LMTO-GW approach has been described in Refs. 28,31-34. It starts from the self-consistent LMTO calculations of the LDA band structure. Such calculations are performed either with the minimal basis set of 
muffin-tin orbitals, i.e., $n s, n p,(n-1) d$ orbitals, or with the extended basis set including higher $d, f$, and $g$ states. ${ }^{31}$ Then the set of Bloch functions employed in many-body calculations is constructed by using the products of intersphere parts of muffin-tin orbitals $\phi_{\mathbf{R} L \nu}$

$$
\beta_{\mathbf{k} i}=\sum_{\mathbf{T}} e^{i \mathbf{k T}} \phi_{\mathbf{R} L \nu}(\mathbf{r}-\mathbf{R}-\mathbf{T}) \phi_{\mathbf{R} l^{\prime} \nu^{\prime}}(\mathbf{r}-\mathbf{R}-\mathbf{T})
$$

where $i=\left\{\mathbf{R} L \nu, \mathbf{R} L^{\prime} \nu^{\prime}\right\}$. The complete set of such products contains linear dependences that are eliminated by performing the diagonalization of the overlap matrix of $\beta_{i}$ functions.

$$
\begin{gathered}
O_{i, j}=\left\langle\beta_{i} \mid \beta_{j}\right\rangle, \\
O z=\lambda z .
\end{gathered}
$$

The resulting orthonormal basis is a linear combination of the product functions

$$
B_{\mathbf{q} i}=\sum_{j} \beta_{\mathbf{q} j} z_{j i}
$$

Using the basis set thus created we calculate the RPA polarization matrix: $:^{32,33}$

$$
\begin{aligned}
\mathbf{P}_{i, j}(\mathbf{q}, \omega)= & \sum_{\sigma, t, \mathbf{k}} \sum_{n}^{o c c} \sum_{n^{\prime}}^{\text {unocc }}\left\langle B_{\mathbf{q} i} \psi_{\mathbf{k}, n} \mid \psi_{\mathbf{k}+\mathbf{q}, n^{\prime}}\right\rangle \\
& \times\left\langle\psi_{\mathbf{k}+\mathbf{q}, n^{\prime}} \mid \psi_{\mathbf{k}, n} B_{\mathbf{q} j}\right\rangle \frac{1}{t \omega-e_{\mathbf{k}+\mathbf{q}, n^{\prime}}+e_{\mathbf{k}, n}+i \delta} .
\end{aligned}
$$

The summation includes terms with $t= \pm$ (electrons and holes) and spin value $\sigma$. We avoid, however, direct use of Eq. (12), making faster computations for the spectral function matrix

$$
\begin{aligned}
S_{i, j}(\mathbf{q}, \omega)= & \sum_{\sigma, t, \mathbf{k}} \sum_{n}^{\text {occ }} \sum_{n^{\prime}}^{\text {unocc }}\left\langle B_{\mathbf{q} i} \psi_{\mathbf{k}, n} \mid \psi_{\mathbf{k}+\mathbf{q}, n^{\prime}}\right\rangle \\
& \times\left\langle\psi_{\mathbf{k}+\mathbf{q}, n^{\prime}} \mid \psi_{\mathbf{k}, n} B_{\mathbf{q} j}\right\rangle \times \delta\left(\omega-e_{\mathbf{k}+\mathbf{q}, n^{\prime}}+e_{\mathbf{k}, n}\right) .
\end{aligned}
$$

This quantity is related to the imaginary part of $P$ as follows

$$
S_{i, j}(\mathbf{q}, \omega)=-\frac{1}{\pi} P_{i, j}(\mathbf{q}, \omega) \operatorname{sgn}(\omega) .
$$

Having obtained $S_{i, j}$ we calculate the real part of $P_{i, j}$ by the Hilbert transform:

$$
\operatorname{Re} P_{i, j}(\mathbf{q}, \omega)=\mathscr{P} \int_{-\infty}^{\infty} d \omega^{\prime} \frac{S_{i, j}\left(\mathbf{q}, \omega^{\prime}\right)}{\omega-\omega^{\prime}} .
$$

Once the polarization matrix is obtained, we use the RPA to evaluate the density-density response function matrix $R$ together with dielectric and inverse dielectric matrices,

$$
R=P+P V R,
$$

$$
\begin{gathered}
\epsilon=1-V P, \\
\epsilon^{-1}=1+V R .
\end{gathered}
$$

The calculations of $R$ in Eq. (16) are performed in the basis of $B_{\mathbf{q} i}$ functions. This is valid also for the Coulomb potential matrix $V$ that is calculated following Ref. 32. By using the $B_{\mathbf{q} i}$ basis we include the local-field effects in the calculations of EELS,

$$
\operatorname{Im} \epsilon^{-1}(\mathbf{q}, \omega)=\operatorname{Im} \sum_{i, j}\left\langle\mathbf{q} \mid B_{\mathbf{q} i}\right\rangle \epsilon_{i, j}^{-1}\left\langle B_{\mathbf{q} j} \mid \mathbf{q}\right\rangle .
$$

Here $\left\langle\mathbf{q} \mid B_{\mathbf{q} i}\right\rangle$ is the Fourier transform of the product basis function.

In order to provide comparison with experimental data, we also calculate the dielectric functions in the plane wave basis $|\mathbf{q}+\mathbf{G}\rangle$. The diagonal elements of the dielectric matrix are expressed for an arbitrary reciprocal lattice vector $\mathbf{G}$ as

$$
\begin{aligned}
\boldsymbol{\epsilon}(\mathbf{q}+\mathbf{G}, \omega)= & 1-\frac{4 \pi}{(\mathbf{q}+\mathbf{G})^{2}} \sum_{i, j}\left\langle\mathbf{q}+\mathbf{G} \mid B_{\mathbf{q} i}\right\rangle P_{i, j} \\
& \times\left\langle B_{\mathbf{q} j} \mid \mathbf{q}+\mathbf{G}\right\rangle .
\end{aligned}
$$

The elements of the inverse dielectric matrix are calculated analogously,

$$
\begin{aligned}
\boldsymbol{\epsilon}^{-1}(\mathbf{q}+\mathbf{G}, \omega)= & 1+\frac{4 \pi}{(\mathbf{q}+\mathbf{G})^{2}} \sum_{i, j}\left\langle\mathbf{q}+\mathbf{G} \mid B_{\mathbf{q} i}\right\rangle R_{i, j} \\
& \times\left\langle B_{\mathbf{q} j} \mid \mathbf{q}+\mathbf{G}\right\rangle .
\end{aligned}
$$

With the inverse dielectric matrix in the basis of productorbitals we calculate the screened Coulomb potential matrix

$$
W(\mathbf{q}, \omega)=\epsilon^{-1}(\mathbf{q}, \omega) V(\mathbf{q})
$$

To finally calculate the self-energy matrix elements we use the equations obtained by replacing the full Green function by the Green function of noninteracting electrons, Ref. 28:

$$
\begin{aligned}
\operatorname{Im} \Sigma_{\mathbf{q}, n}(\omega)= & \sum_{\mathbf{k}} \sum_{n^{\prime}}^{o c c} \sum_{i, j}\left\langle\psi_{\mathbf{q}, n} \psi_{\mathbf{k}-\mathbf{q}, n^{\prime}} \mid B_{\mathbf{k} i}\right\rangle \\
& \times \operatorname{Im} W_{i, j}\left(\mathbf{k}, e_{\mathbf{k}-\mathbf{q}, n^{\prime}}-\omega\right) \\
& \times\left\langle B_{\mathbf{k} j} \mid \psi_{\mathbf{k}-\mathbf{q}, n^{\prime}} \psi_{\mathbf{q}, n}\right\rangle \theta\left(e_{\mathbf{k}-\mathbf{q}, n^{\prime}}-\omega\right),
\end{aligned}
$$

when $\omega \leqslant \mu$ and

$$
\begin{aligned}
\operatorname{Im} \sum_{\mathbf{q}, n}(\omega)= & -\sum_{\mathbf{k}} \sum_{n^{\prime}}^{\text {unocc }} \sum_{i, j}\left\langle\psi_{\mathbf{q}, n} \psi_{\mathbf{k}-\mathbf{q}, n^{\prime}} \mid B_{\mathbf{k} i}\right\rangle \\
& \times \operatorname{Im} W_{i, j}\left(\mathbf{k}, \omega-e_{\mathbf{k}-\mathbf{q}, n^{\prime}}\right) \\
& \times\left\langle B_{\mathbf{k} j} \mid \psi_{\mathbf{k}-\mathbf{q}, n^{\prime}} \psi_{\mathbf{q}, n}\right\rangle \theta\left(\omega-e_{\mathbf{k}-\mathbf{q}, n^{\prime}}\right) .
\end{aligned}
$$

when $\omega>\mu$. 

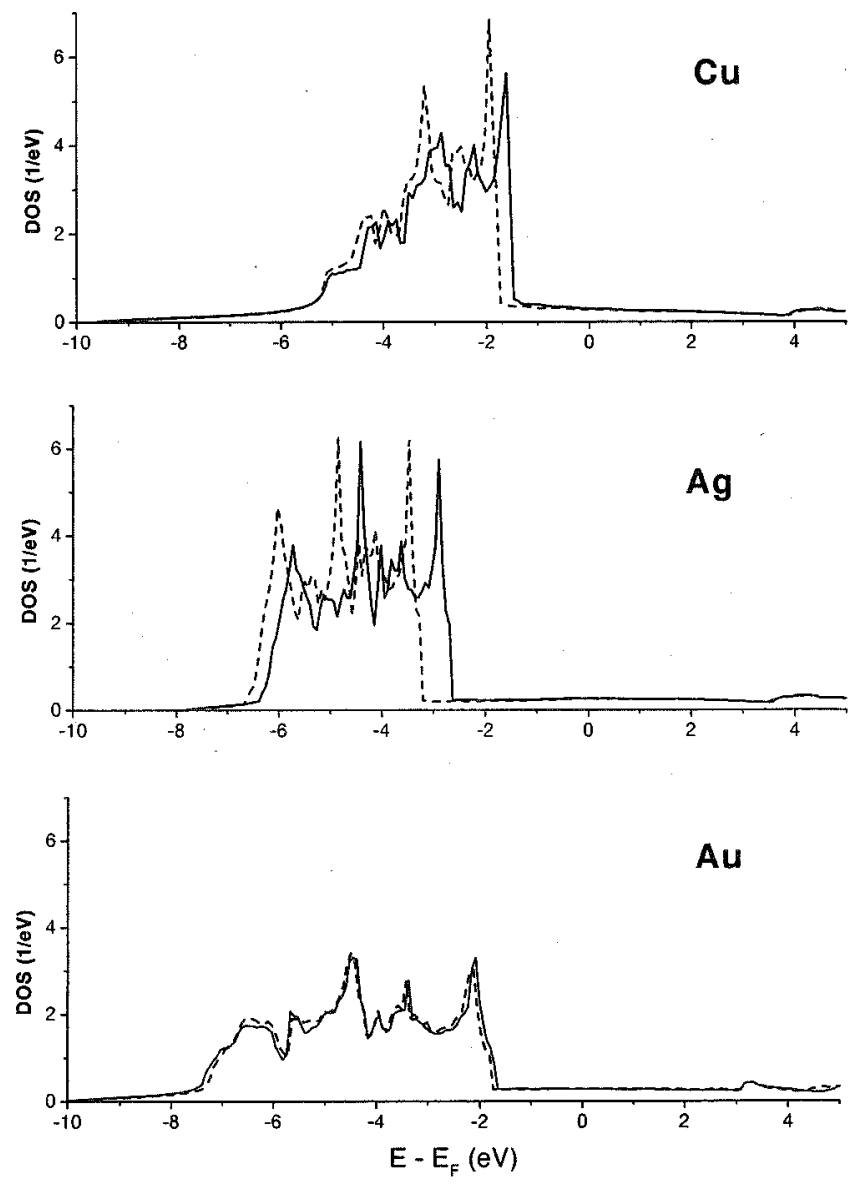

FIG. 1. Self-consistent total densities of states (DOS) in $\mathrm{Cu}, \mathrm{Ag}$, and $\mathrm{Au}$ calculated by the standard LMTO method (solid lines). Dashed lines show the DOS computed with the modified value of a continuous principal quantum number, $P(d)=3.85(\mathrm{Cu}), 4.85(\mathrm{Ag})$, $5.87(\mathrm{Au})$

Practically, instead of full screened potential we use its correlation part $W^{c}=W-V$ that only contributes to the imaginary part of the self-energy. ${ }^{27,33}$

We calculated the LDA band structures of noble metals by using the tight-binding version of the LMTO method, ${ }^{35,36}$ with the minimum basis set that includes the $n s, n p,(n$ $-1) d$ states. To check the convergence with respect to the extension of a muffin-tin basis set, we also performed for $\mathrm{Cu}$ the evaluations of the band structure, dielectric functions and lifetimes with the extended basis set that includes highenergy $n d$ and $4 f$ orbitals. For the energy interval $0-5 \mathrm{eV}$ above the Fermi level that is usually studied in experiments, such extension of the basis set does not change the calculated results noticeably. The convergence of the results with increase of the number of $\mathbf{k}$ vectors has also been verified. The conclusion is that the minimal number of $\mathbf{k}$ vectors needed is about 250 per irreducible part of the Brillouin zone ( 8000 per Brillouin zone). For the energy interval discussed the calculations with higher numbers of $\mathbf{k}$ vectors produce no important changes either in band structures or in dielectric functions. To avoid numerical complications relating to the singularity of the Coulomb potential at $\mathbf{q}=0$, the calculations of dielectric functions were performed at $\mathbf{q}=(2 \pi / a)$

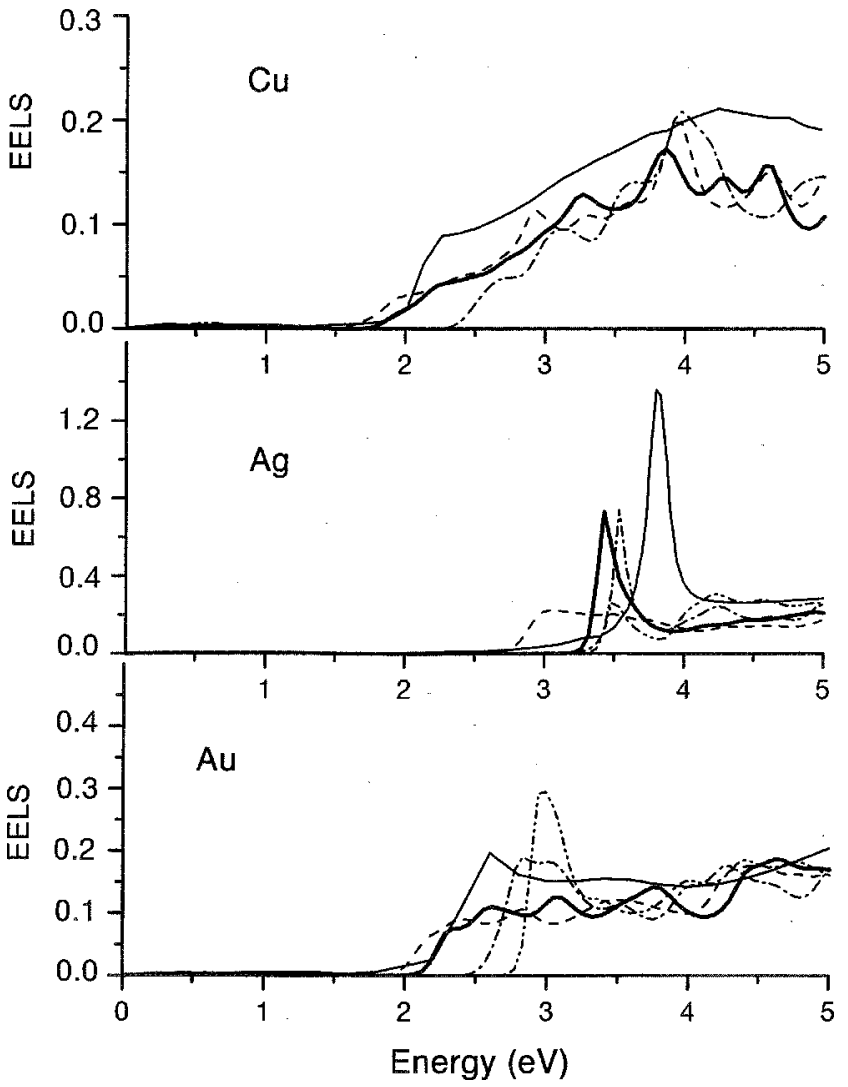

FIG. 2. Experimental (thin solid lines) and "the best" calculated (thick solid line) EELS for $\mathrm{Cu}, \mathrm{Ag}$, and $\mathrm{Au}$. The best calculated EELS correspond to $P(d)=3.85(\mathrm{Cu}), 4.85(\mathrm{Ag})$, and $5.87(\mathrm{Au})$. EELS obtained from the self-consistent band structures are shown by dashed lines, the $P(d)$ parameter is equal to $3.87(\mathrm{Cu}), 4.90$ $(\mathrm{Ag})$, and $5.89(\mathrm{Au})$. Shown by dash-dotted lines are EELS calculated with $P(d)=3.77(\mathrm{Cu}), 4.80(\mathrm{Ag})$ and $5.79(\mathrm{Au})$. Dash-dotdotted lines present the calculated EELS with $P(d)=4.75(\mathrm{Ag})$ and $P(d)=5.74(\mathrm{Au})$.

$\times(0.05,0.05,0.05)$ that is the shortest in our set of $\mathbf{k}$ vectors. Creating the $B_{\mathbf{q} i}$-basis vectors, all possible products of muffin-tin orbitals within the minimal basis set $(s \times s$, $s \times p, s \times d, p \times p, p \times d)$ have been used. In the calculations of the spectral function matrix the $\delta$ functions were replaced with a gaussian

$$
\delta(x) \rightarrow \frac{1}{\Gamma \sqrt{\pi}} e^{-x^{2} / \Gamma^{2}} .
$$

The broadening factor $\Gamma$ employed was equal to $0.136 \mathrm{eV}$ (0.005 Hartree a.u.).

\section{DIELECTRIC FUNCTIONS OF NOBLE METALS}

The band structures of noble metals calculated by the LMTO method within LDA do not differ essentially from earlier results-see, e.g., Ref. 37. In Fig. 1 we show the density of states (DOS) of $\mathrm{Cu}, \mathrm{Ag}$, and $\mathrm{Au}$. The ingredients of the DOS are the low DOS of $n s, n p$ states extending from $-10 \mathrm{eV}$ to $30 \mathrm{eV}$ and the high $(n-1) d$ DOS around -7 to $-1 \mathrm{eV}$, all the energies being relative to the Fermi level. 

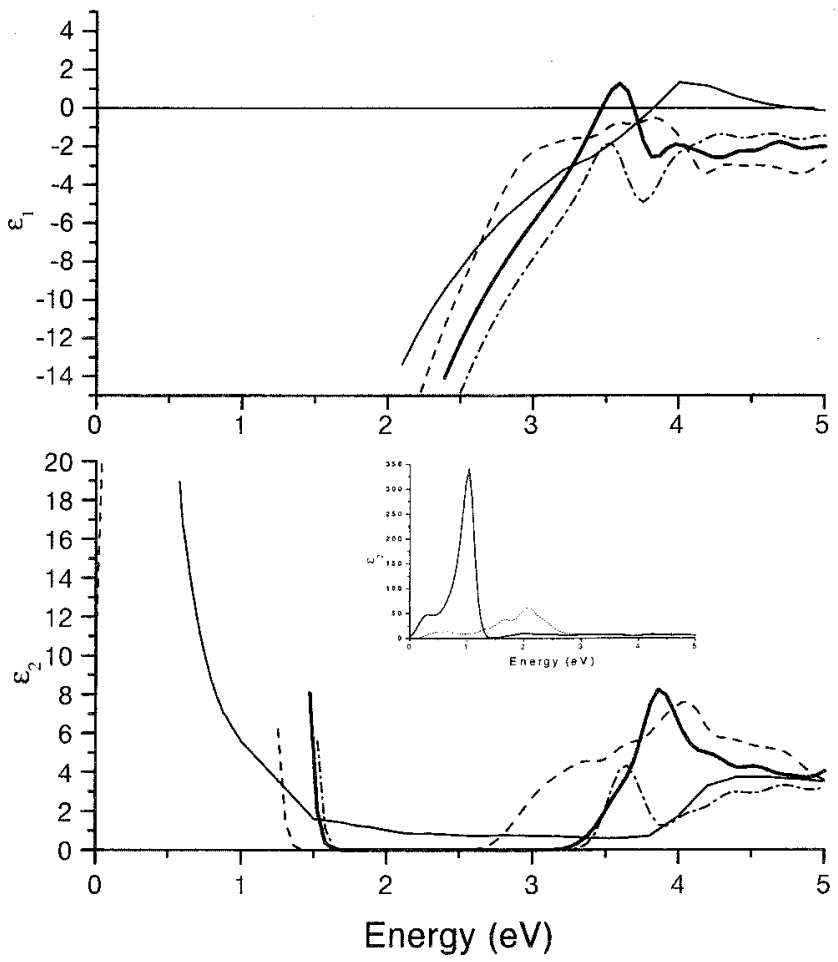

FIG. 3. Real and imaginary part of the dielectric function calculated for Ag at $\mathbf{q}=(2 \pi / a)(0.05,0.05,0.05)$. Thin solid lines represent experimental data, while thick solid lines correspond to "the best" calculated data with $P(d)=4.85$. Shown by dashed lines are the results for the self-consistent band structure with $P(d)=4.87$ whereas dash-dotted lines correspond to $P(d)=4.77$. The inset shows the calculated imaginary part for $\mathbf{q}=(2 \pi / a)$ $\times(0.05,0.05,0.05) \quad($ solid line) and $\mathbf{q}=(2 \pi / a)(0.1,0.1,0.1)$ (dashed line).

The $4 d$ states of Ag begin with an abrupt change of DOS at the energy of $-2.7 \mathrm{eV}$. The energy interval between this threshold and the Fermi level is small compared to experimental photoemission data, $3.8 \mathrm{eV}^{38}$ For $\mathrm{Cu}$ and Au the calculated $d$-band threshold energy is equal to $1.5 \mathrm{eV}$ and 1.7 $\mathrm{eV}$, respectively, compared to photoemission data $2.0 \mathrm{eV}$ and $2.5 \mathrm{eV}^{38}$ So the calculated energy of $d$ states is too high, especially in the case of Ag. This drawback of the LDA may explain why the plasmon peak in the EELS of $\mathrm{Ag}$ is not reproduced in pseudopotential plane-wave LDA calculations. ${ }^{24}$ The analogous effect takes place in the conventional LDA calculations for Zn (Ref. 39) where the calculated EELS differs essentially from the threshold line shape observed in the experimental spectroscopic data.

Hence, in order to better describe dielectric functions, it is desirable to improve the LDA band structures. In Ref. 39 this has been done by changing the $\alpha$ coefficient in Slater's approximation for the exchange-correlation potential. In this paper we retain a nonempirical approach to the exchangecorrelation potential, and modify the LDA band structure by changing the continuous principal quantum number of $d$ states, ${ }^{36}$

$$
P_{l}=0.5+\arctan \left[D_{l}\left(E_{\nu}\right)\right] / \pi+n .
$$

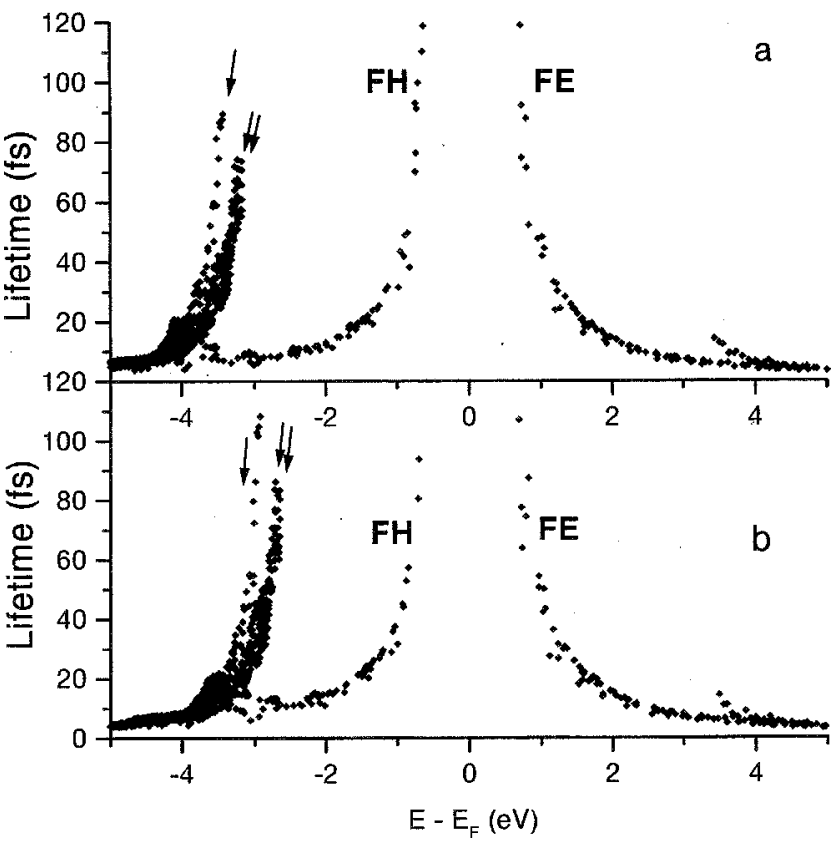

FIG. 4. The calculated quasiparticle lifetimes for Ag with the corrected LDA band structure at $P(d)=4.85$ (panel a) and with the self-consistent LDA band structure at $P(d)=4.90$ (panel b). FH (FE) indicates the hole (electron) lifetimes in the $s-p$ band. One and two arrows depict the hole lifetimes in the upper $d$ bands.

Here $D_{l}\left(E_{v}\right)$ is the logarithmic derivative of the basis muffin-tin orbital at the atomic sphere, calculated at the energy $E_{\nu}$ that is usually chosen to be equal to the center of gravity of the occupied bands. The value of the $P_{l}$ parameter is connected with the shape of the muffin-tin orbital: the more localized the radial part of the orbital the smaller it is. So this value is determined by the charge distribution inside the atomic sphere. The parameter $P_{l}$ is used to construct the LMTO TB (tight-binding) Hamiltonian matrix ${ }^{36}$ and in selfconsistent calculations it is recalculated at each iteration step.

Expecting that with the decrease of $P_{l}$ of $d$ states, $P(d)$, the magnitude of exchange-correlation energy would increase thus shifting $d$ bands to lower energies, we performed the calculations of band structures and dielectric functions with $P(d)$ decreased from its self-consistent value. It follows from the dashed curves in Fig. 1 that indeed the $d$ bands of noble metals are shifted to negative energies without changes in the shape of DOS, thus improving agreement with photoemission measurements. Such calculations are not exactly self-consistent: after self-consistency is achieved in a standard way, i.e., with finally self-consistent $P_{l}$ values, we perform only one iteration step with the changed $P(d)$. If the self-consistency process with the changed and fixed $P(d)$ were continued, the final results would be almost indistinguishable from the self-consistent results. So the truly selfconsistent LMTO calculations are insensitive to small changes in $P$.

Comparing with experimental data we focus on the energy interval between 0 and $5 \mathrm{eV}$, because it is for this region that the experimental and theoretical lifetime researches are usually performed. The effect of the $P(d)$ parameter on the electron energy loss spectra is illustrated in Fig. 2. In com- 


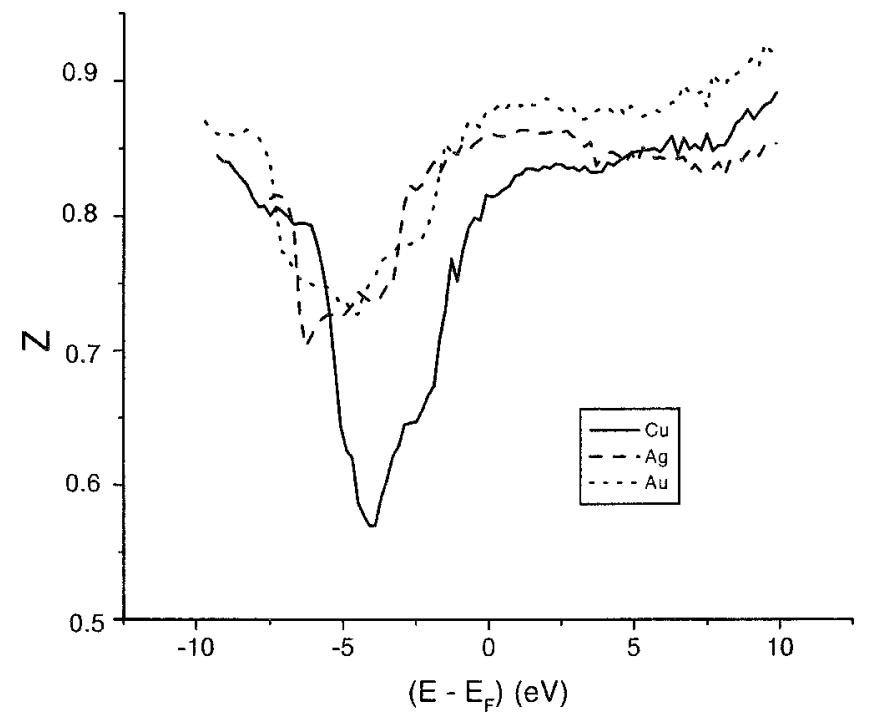

FIG. 5. The energy dependence of the averaged renormalization factor $\mathrm{Z}$ for $\mathrm{Cu}, \mathrm{Ag}$, and $\mathrm{Au}$.

parison with experimental data, ${ }^{40}$ certain precautions should be taken relating to the nonzero value of the momentum $\mathbf{q}$ used in our calculations, whereas the optical data refer to $\mathbf{q}$ $=0$. The calculated EELS are typically lower than the optical data. This is compatible with the results of Ref. 20 that also show the decrease of the calculated EELS with increase of q. Generally with the decrease of $P(d)$ the threshold of interband optical absorption shifts to higher energy. For $\mathrm{Cu}$ the calculated onset energy of the interband transition appears to be in very close agreement with optical measurements at $P(d) \simeq 3.85$. With further decrease of $P(d)$ the agreement becomes worse. For Ag it follows from Fig. 2 that with the $P(d)$ value being in the interval between 4.85 and 4.80 a plasmon absorption peak emerges in the vicinity of $3.5 \mathrm{eV}$, while with lower values of $P(d)$ it disappears. For Au good agreement between the calculated and experimental data for the threshold energy is achieved at $P(d) \simeq 5.87$, which is very close to the self-consistent value of 5.89. A very damped plasmon emerges at $P(d)=5.75$. However, the last calculations have no real physical meaning, because at such $P(d)$ the energy of $d$ bands is too low compared with experimental data.

Figure 3 shows the changes of dielectric functions of $\mathrm{Ag}$ with changes in the $P(d)$ parameter. In the results for $\epsilon_{2}$ two sources of disagreement between the calculated and optical data become evident. The first one is observed at approximately $1 \mathrm{eV}$ where a large difference appears between the experimental and calculated edge of the intraband absorption. This discrepancy is most probably related not to a drawback of the LDA calculations but rather to the nonzero value of $\mathbf{q}$ momentum used for comparisons with experimental data. In order to confirm this we show in the inset to Fig. 3 the graphs of $\epsilon_{2}$ of $\mathrm{Ag}$ with the values of $\mathbf{q}$ equal to $(2 \pi / a)(0.05,0.05,0.05)$ and $(2 \pi / a)(0.1,0.1,0.1)$. The differences between these results are drastic: with the decrease of momentum the maximum of $\epsilon_{2}$ increases by a factor of 7 and shifts by $1 \mathrm{eV}$ to zero energy. Hence one can expect better agreement with optical data using very small values of

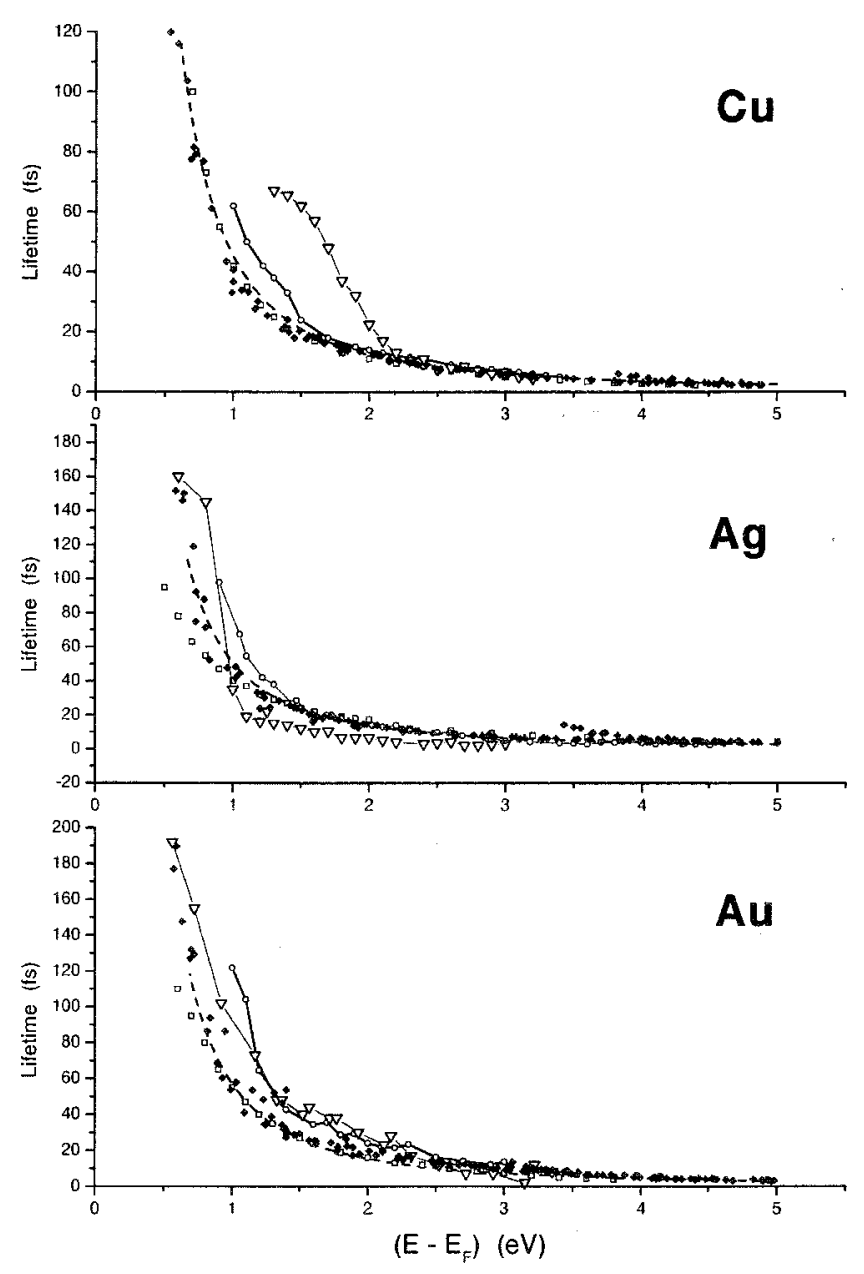

FIG. 6. The calculated and experimental data on electron lifetimes. Solid diamonds show our LMTO results; open squares represent the calculated data of Refs. 21 and 23 along the $(1,1,0)$ direction. Open circles depict the computed data of Refs. 22 and 25 for $\mathrm{Cu}$ and $\mathrm{Au}$, respectively, and our new pseudopotential planewave calculation results for $\mathrm{Ag}$. Open triangles are experimental data of Refs. 6,11 , and 41 along the $(1,1,0)$ direction. Dashed lines represent the results of FEG theory with the adjusted $r_{s}$ parameter, see text.

q. The second drawback, similar to that of EELS, is a too low energy for the onset of interband transitions that take place between 3 and $4 \mathrm{eV}$. Decreasing $P(d)$ leads to much better agreement with the experimental data. Through the Hilbert transform it changes the $\epsilon_{1}$ value that becomes zero at the energy of $3.5 \mathrm{eV}$ for $P(d)$ values in the interval 4.80 4.85. The calculated $\epsilon_{2}$ turns out to be small at this energy, so that the peak of plasmon absorption emerges. With $P(d)$ below this interval $\epsilon_{1}$ is again negative, so the plasmon disappears. One further conclusion is that of the two components of $\epsilon$ that govern the emergence of plasmon, the $\epsilon_{1}$ value is the more important. In fact, $\epsilon_{2}$ is nearly the same: either plasmon exists at $P(d)=4.80$ or disappears at $P(d)$ $=4.75$.

The changes in the dielectric functions of $\mathrm{Cu}$ and $\mathrm{Au}$ with changing $P(d)$ are similar to those of Ag. However, there is an important difference: the $\epsilon_{1}$ curve of $\mathrm{Cu}$ does not pass 


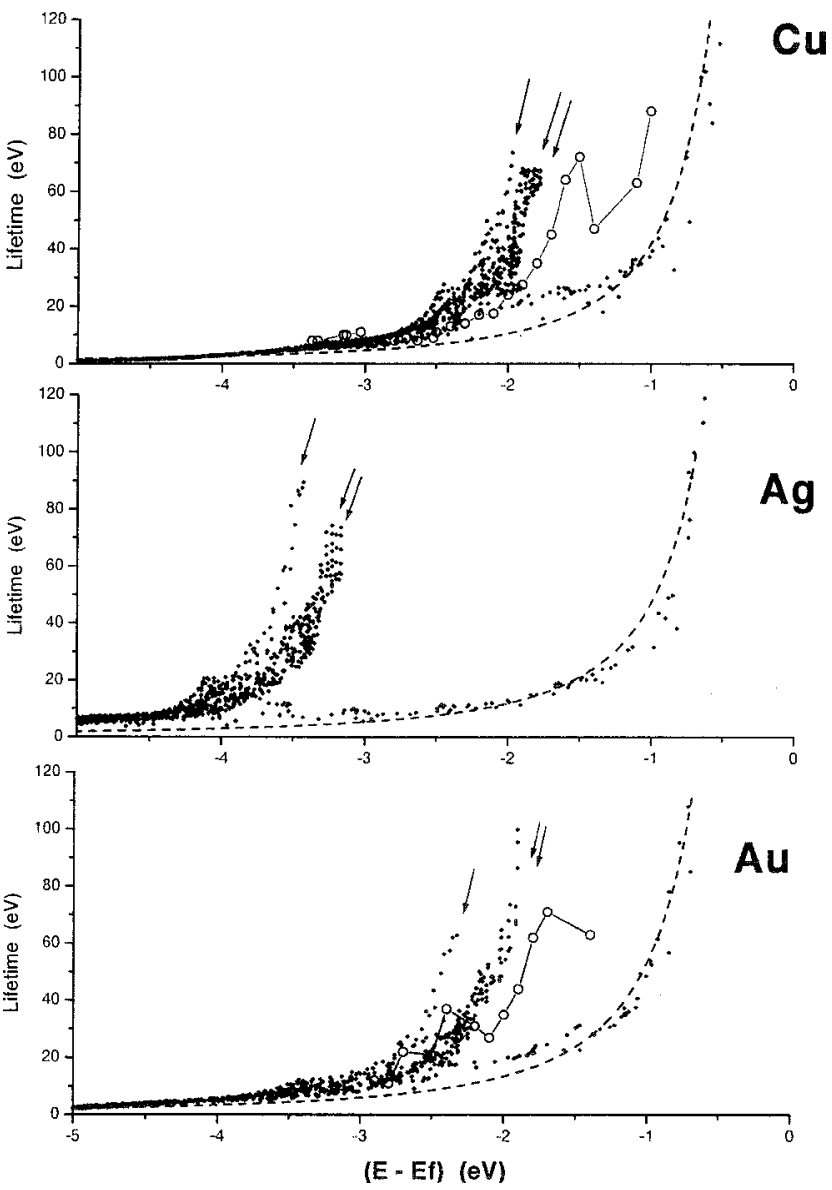

FIG. 7. The calculated hole lifetimes for noble metals. Solid diamonds represent our results; open circles depict the calculated data of Ref. 25. Dashed lines indicate the results of FEG theory with the adjusted $r_{s}$ parameter, see text.

through zero, while for Au it becomes zero only at such small values of $P(d)$ as to have no real physical meaning.

\section{QUASIPARTICLE LIFETIMES IN NOBLE METALS}

Changing the value of continuous principal quantum number one can adjust the threshold of $d$ bands to experimental photoemission data and hence avoid some drawbacks pertinent to the LDA band structures. To verify if the quasiparticle lifetimes depend on such modifications of the band structure we performed the calculations for Ag with the selfconsistent value of the parameter $P(d)=4.90$ and with $P(d)=4.85$. The results are shown in Fig. 4. One can distinguish freelike electrons (FE) and holes $(\mathrm{FH})$; these are quasiparticles in the $s, p$ bands. A further type of holes belongs to the arrays marked by one and two arrows; they correspond to the excitations in $4 d$ bands.

The FE and FH lifetime values for "the best" band structure in the panel $a$ do not differ much from the selfconsistent data in the panel $b$. This is also valid for $d$ holes, the data in the panel $a$ being mainly shifted to lower energies in accordance with the shifts of the LDA eigenenergies. So one may conclude that the drawbacks in the LDA band structures discussed above do not lead to qualitative changes in

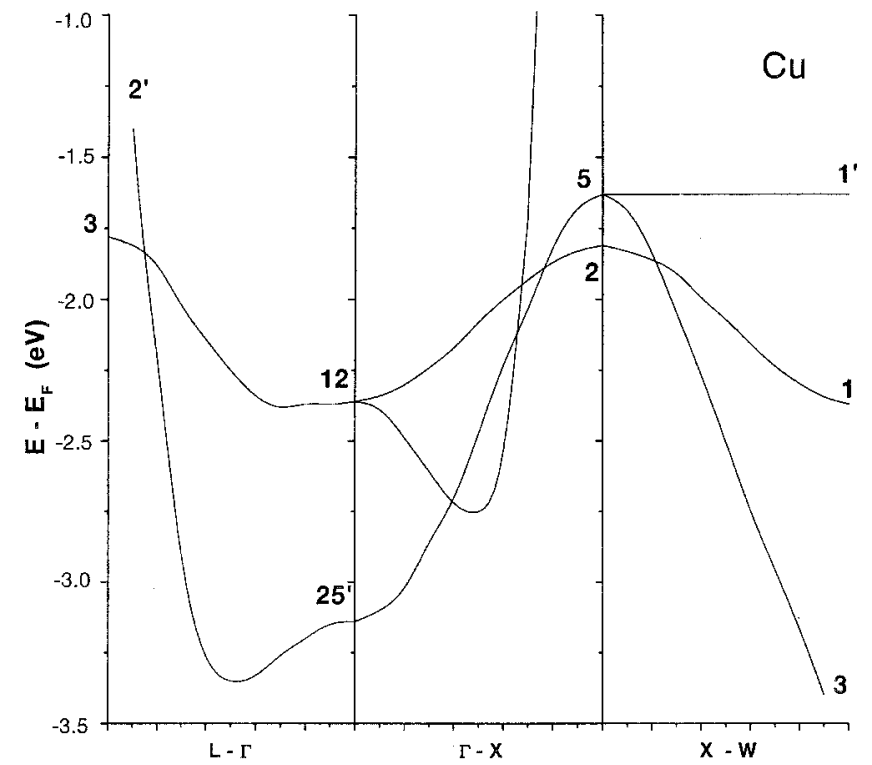

FIG. 8. The energy band structure of the upper $d$ states of $\mathrm{Cu}$.

quasiparticle lifetimes. In particular, the lifetimes of $\mathrm{Ag}$ do not depend essentially on the presence or absence of plasmon absorption. In order to reveal the origin of such stability, we performed for Ag the calculations of EELS at higher values of $\mathbf{q}$ momentum. These calculations show that the differences between the EELS obtained from the conventional and from the corrected LDA band structure are noticeable only at small momenta, and with increasing $\mathbf{q}$ the intensity of the plasmon rapidly decreases. As follows from Eq. (23) the selfenergy corrections are determined by the screened potential at all the values of momentum, so the inaccuracies in LDA band structures at small $\mathbf{q}$ appear to be unimportant.

The averaged renormalization factor $\mathrm{Z}$ of $\mathrm{Cu}, \mathrm{Ag}$, and $\mathrm{Au}$ is given in Fig. 5. In all the metals of interest the calculated $Z$ is quite far from the 1.0 used in the energy shell approximation. The values of $Z$ for electron excitations are in good agreement with those obtained in the FEG model. ${ }^{32,46}$ For $d$-hole excitations the renormalization constant $Z$ varies between 0.57 and 0.77 . So for these excitations the use of the true $Z$ values is especially important.

Below we present and discuss the best data obtained by using the corrected LDA band structures and with the calculated values of $Z$. In Fig. 6 we compare our calculated electron lifetimes with the lifetimes of Ref. 21 and 23 that were obtained within the LDA pseudopotential plane wave approach. For Ag we also show our new results calculated within the energy shell approximation using the pseudopotential approach. The quasiparticle energies of Dyson equation were calculated in Refs. 21 and 23 by searching for the poles of a Green function near the real axis of energy. So, in the calculations of Refs. 21 and 23 the renormalization factor has implicitly been taken into account. In spite of great differences in the methods of calculation, very good agreement is observed for $\mathrm{Cu}$ between our data and the results of Refs. 21 and 23; at energies above $1 \mathrm{eV}$ the agreement is also good for $\mathrm{Ag}$ and $\mathrm{Au}$. Slightly worse is the accord between our data and the averaged lifetime data of Ref. 22 calculated within the energy shell approximation, i.e., $Z=1$. We also show the 


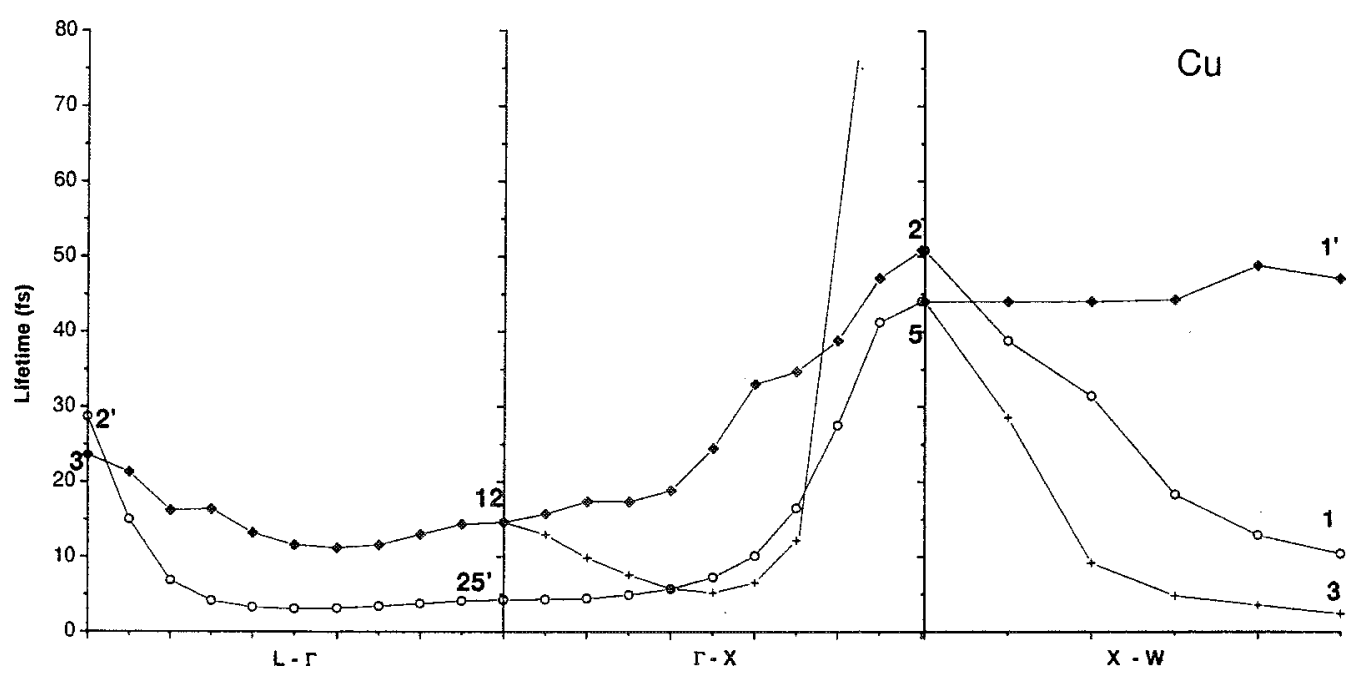

FIG. 9. The $d$-hole lifetime band structure of $\mathrm{Cu}$.

experimental data on the lifetimes for $\mathrm{Cu}$ from Ref. 6, $\mathrm{Ag}$ from Ref. 41 and Au from Ref. 11. Unlike the calculated data, the experimental results display a sudden increase at the energy below $2 \mathrm{eV}$ for $\mathrm{Cu}$ and $1 \mathrm{eV}$ for Au. The origin of disagreement between calculated and experimental data was discussed in Ref. 42. The authors attribute the disagreement to excitonic effects that are not taken into account in the model of hot electron damping employed. Other mechanisms discussed that can influence the lifetimes at these energies are electron-phonon interactions and electron transport. ${ }^{11}$

In Figs. 4 and 6 we present all the calculated lifetime values, and these data show that the dispersion of the electron excitation lifetimes with respect to the momentum appear to be small. A smooth dispersionless decrease of the electron lifetime with increase of energy is an intrinsic feature of the free-electron gas model. The attempts to describe the lifetimes of the $s, p$ electrons in noble metals within the FEG model lead at low energy to rather unsatisfactory results. ${ }^{23}$ The origin of this failure is evident: the electron density parameter $r_{s}$ of the FEG is not determined only by the density of the $s, p$ electrons. The $d$ electrons also contrib- ute to $r_{s}$, and in general this parameter is unknown. Here we find the $r_{s}$ value by adjusting the results of the FEG theory to the lifetimes of our LMTO-GW calculations. The $r_{s}$ values obtained in such a way are equal to 2.1,2.0, and 1.9 for $\mathrm{Cu}$, $\mathrm{Ag}$, and $\mathrm{Au}$, respectively. At such values of $r_{s}$ good agreement is observed between the results of the LMTO-GW calculation and the FEG model.

The hole excitations in $\mathrm{Cu}, \mathrm{Ag}$, and $\mathrm{Au}$ appear to represent a very different case, as follows from Fig. 7. The lifetimes of holes in $s, p$ bands, whose energies are above $-1.5 \mathrm{eV}$, are in good agreement with the FEG theory when the $r_{s}$ parameter is adjusted to electron excitations. Big deviations from the FEG model are observed for holes in the upper $d$ bands where the calculated lifetimes are factors of 8-10 longer than the FEG values. In particular, long lifetimes are obtained for the holes in states near the $X_{2}-\Gamma_{12}$ direction, marked by one arrow in Figs. 4 and 7. The second group of such holes belongs to states close to the $W_{1},-X_{5}-L_{3}-\Gamma_{12}$ directions; they are marked by two arrows. The energy dispersion curves for the states with long hole lifetimes are given for $\mathrm{Cu}$ in Fig. 8. For $\mathrm{Ag}$ and $\mathrm{Au}$ such band

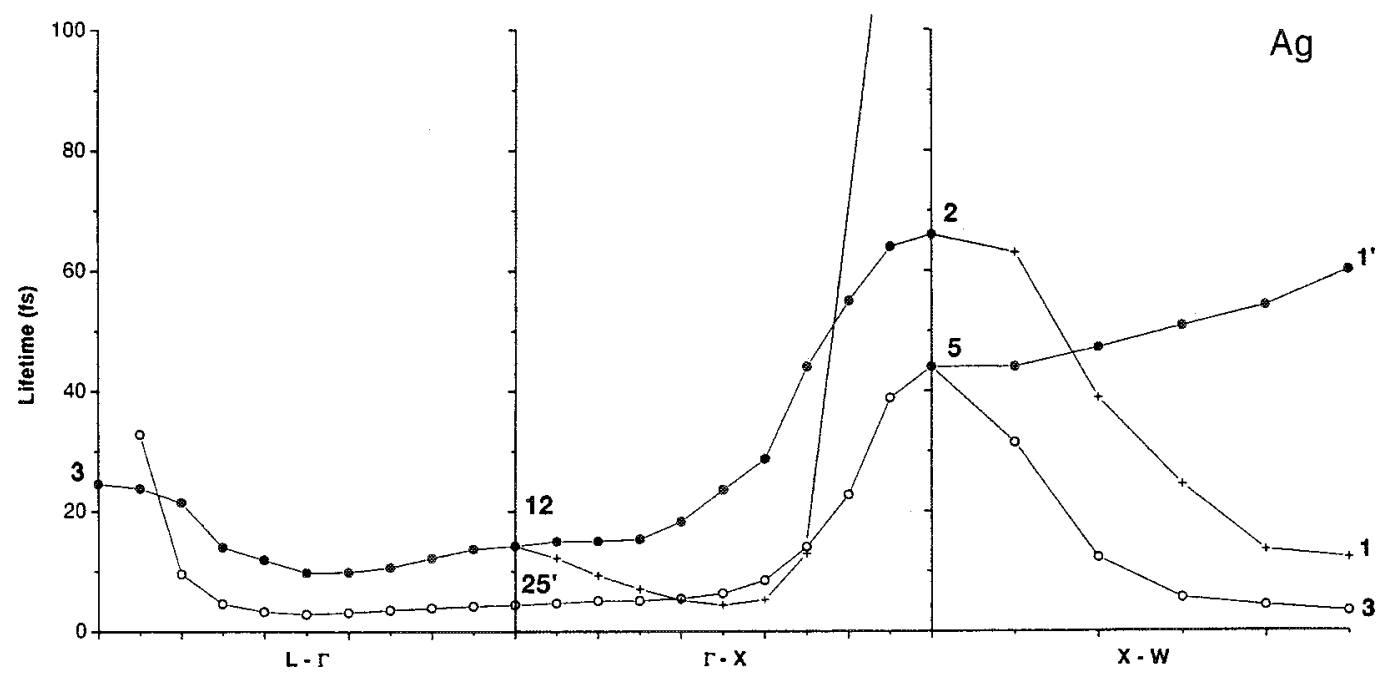

FIG. 10. The $d$-hole lifetime band structure of Ag. 


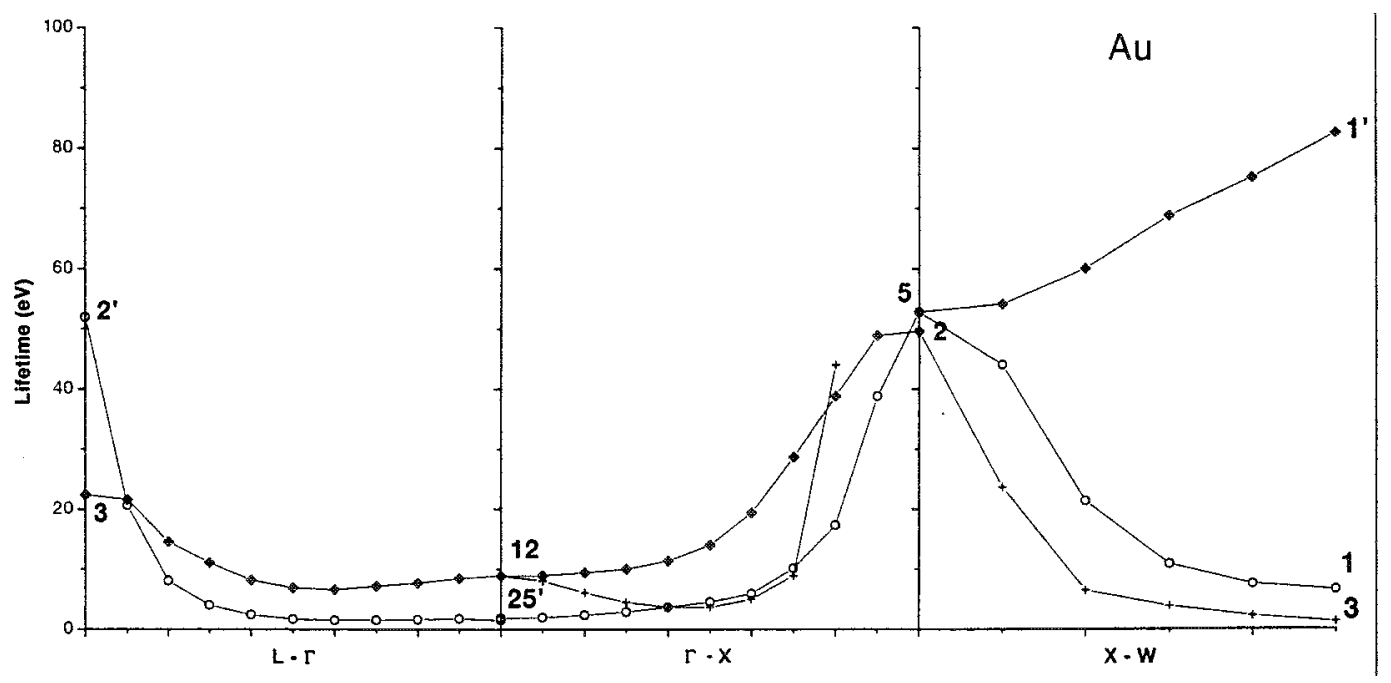

FIG. 11. The $d$-hole lifetime band structure of Au.

structures are similar to that of $\mathrm{Cu}$, differing mainly by a uniform energy shift.

A detailed picture of the $d$-hole lifetimes is provided by Figs. 9-11. Those show the "lifetime band structures" for some of the energy states plotted in Fig. 8. The long hole lifetimes at the top of $d$ bands are qualitatively explained by different symmetry of these states and of the $s, p$ states at higher energy that provide channels for the damping of the $d$ holes. ${ }^{43}$ Due to the symmetry, the oscillator strengths of the transitions between the $d$-hole state and the $n s, n p$ states are small. It follows from Fig. 7 that with the decrease of the energy of a $d$ hole its lifetime generally rapidly decreases. This can be explained by a rapid increase of the number of higher $d$ states that have large oscillator strengths with the $d$-hole states. Figures $9-11$ show that this appears to be also valid when one follows the lifetime changes along the symmetry directions of the Brillouin zone. One finds, however, some exceptions. The examples are $X_{2}$ and $X_{5}$ states: for $\mathrm{Ag}$ $e_{L D A}\left(X_{2}\right)<e_{L D A}\left(X_{5}\right)$, but $\tau\left(X_{2}\right)>\tau\left(X_{5}\right)$. One more exclusion is the $X_{5}-W_{1}$, band: this energy band is very flat, and the changes of hole lifetime in this band are determined by the changes of oscillator strength.

Unlike the case of electron excitations, the amount of available experimental data on hole dynamics in the noble metals is very limited. Recently the dynamics of $d$ holes in $\mathrm{Cu}$ have been deduced from TR-2PPS measurements ${ }^{44}$ and from one-photon photoemission experiments. ${ }^{45}$ Knoesel et $a l .{ }^{8}$ estimated the lifetime of a hole in the upper $d$ band as $\tau=35$ fs, while Petek et al. ${ }^{44}$ obtained for the $X_{5}$ point a value of $24 \mathrm{fs}$. These lifetimes are shorter than our theoretical values (45 fs, Fig. 9) by factors of 1.2-1.8. The evaluations performed in Ref. 45 give the lifetime value as $11.7 \mathrm{fs}$ at the excitation energy $3 \mathrm{eV}$ that is twice our result, see Fig. 7. The same evaluations produce at the energy $2 \mathrm{eV}$ a lifetime value equal to $26 \mathrm{fs}$, in good ageement with our data. So the discrepancy between experimental and theoretical data for hole excitations can be significant. However, the conclusions about long lifetimes of holes at the top of $d$ bands, ${ }^{45}$ rapidly decreasing with the reduction in energy, are confirmed by the calculations.
The effect of increasing the hole lifetimes at the top $d$ bands becomes more pronounced if one considers the ratio of the hole lifetime $\tau(h)$ to the electron lifetime $\tau(e)$ at the same magnitude of energy. To provide a clearer insight into the hole decay mechanisms we calculated the values of both electron and hole lifetimes averaged over wave vectors for each fixed energy. In Figs. 12-14 we show this ratio obtained from our LMTO-GW evaluations for $\mathrm{Cu}, \mathrm{Ag}$, and $\mathrm{Au}$ and compare it with the results of pseudopotential GW (Ref. 25) and FEG calculations. As follows from Figs. 12 and 14 the curves obtained in Ref. 25 are qualitatively similar to our $\tau(e) / \tau(h)$ dependence. Both these curves show a sharp peak at the energy of the top $d$ bands, which are characterized by very long lifetimes. The main quantitative difference is the shifted position of our curve to higher energies. A further

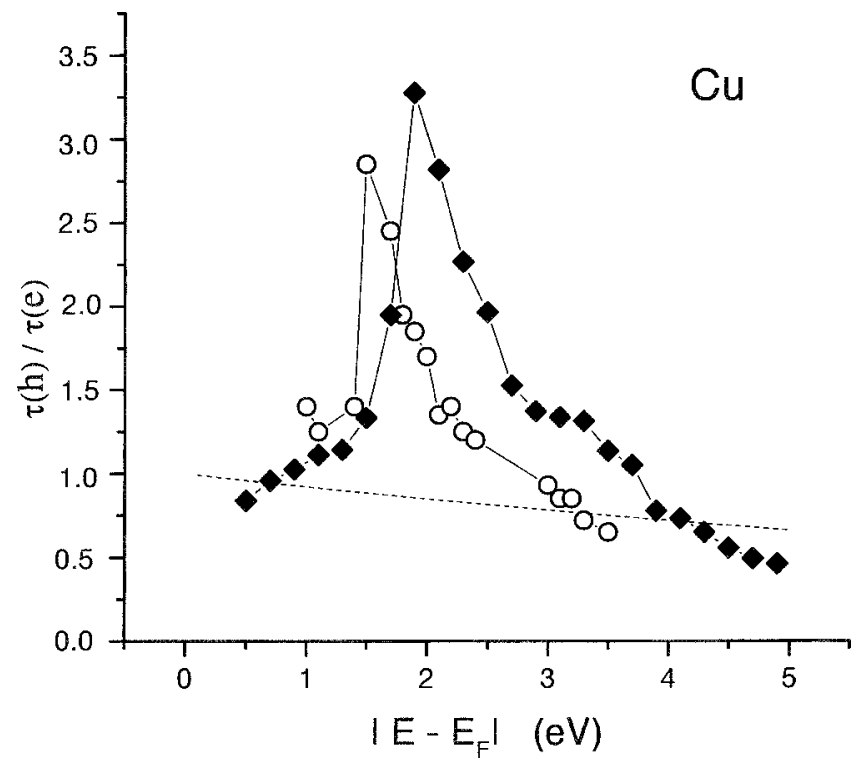

FIG. 12. Ratio between the hole and electron lifetimes in $\mathrm{Cu}$ obtained from our LMTO-GW evaluations (solid diamonds), pseudopotential plane-wave GW calculations (Ref. 25) (open circles) and FEG theory with $r_{s}=2.1$ (dashed line). 


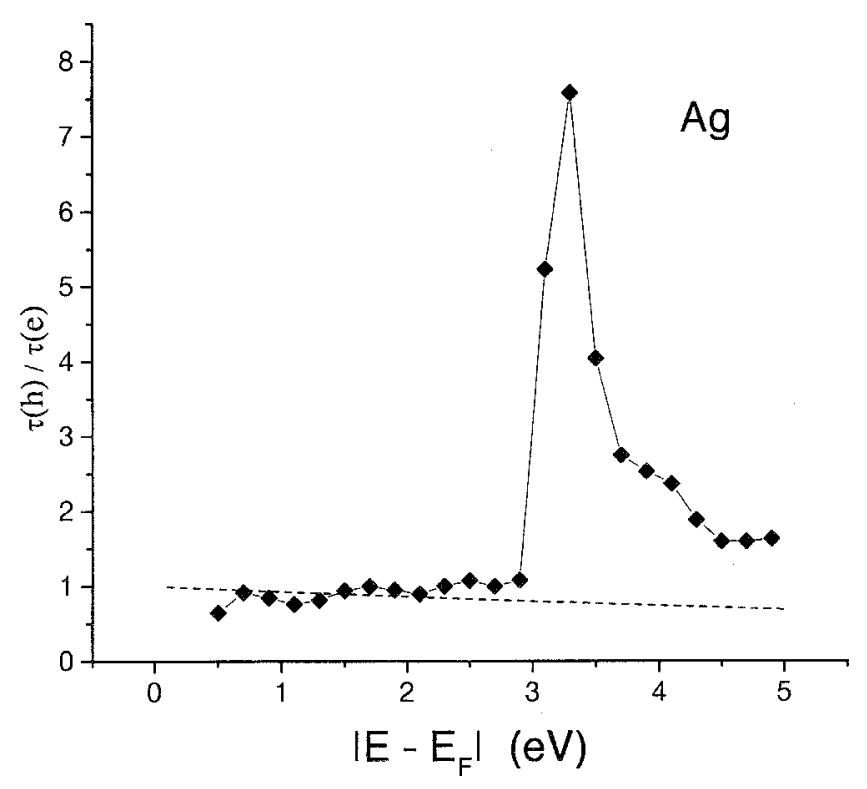

FIG. 13. Ratio between the hole and electron lifetimes in $\mathrm{Ag}$ according to our LMTO-GW evaluations (solid diamonds) and FEG theory with $r_{s}=2.0$ (dashed line).

difference is observed for energies smaller than $1 \mathrm{eV}$. However, the most striking discrepancy is observed between the results based on band structure and FEG results. The latter exhibit completely structureless behavior that is a direct consequence of the absence of $d$ states in the free electron gas model.

\section{CONCLUSIONS}

The numerical calculations performed with varying band structures show that the energy of the $d$ bands is very important for a correct description of the dielectric functions of noble metals for small momenta. Within the LMTO method, the changes of the $P(d)$ parameter seems to be a sufficiently good tool to make corrections to the LDA band structure. For $\mathrm{Cu}$ and $\mathrm{Au}$ only a small decrease of the principal quantum number $P(d)$ appears to be necessary to adjust the calculated energy of the onset of interband transitions to the experimental EELS data. With slight changes of $P(d)$ the plasmon absorption peak emerges in the calculated EELS of Ag.

The evaluations performed with the conventional and corrected self-consistent LDA band structures lead to a very optimistic conclusion that the LDA drawbacks in the descriptions of the band structures do not influence qualitatively the quasiparticle lifetimes. The quantitative differences are mainly due to the shifts of the $d$ bands to lower energies that does not influence the hole and electron lifetimes in the $s, p$ bands. More important are the calculations of the renormalization factor $Z$ : taking $Z$ into account brings the calculated electron lifetimes into close agreement with the results of a direct search of the quasiparticle energies.

The calculations of the electron lifetimes in $s, p$ states

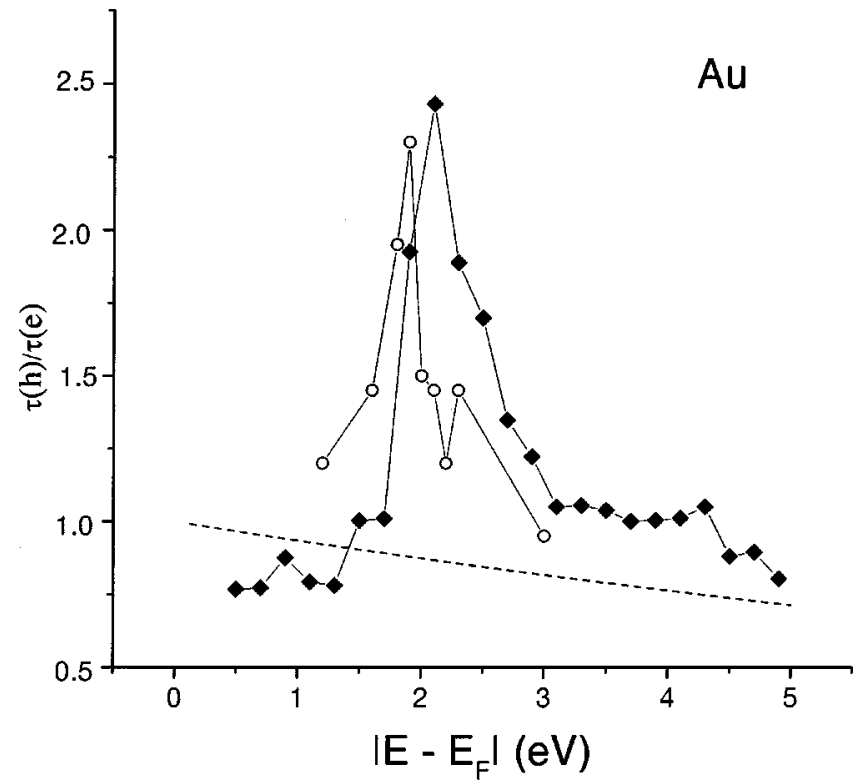

FIG. 14. Ratio between the hole and electron lifetimes in $\mathrm{Au}$ according to our LMTO-GW evaluations (solid diamonds), pseudopotential plane-wave GW calculations (Ref 25) (open circles) and FEG theory with $r_{s}=1.9$ (thick solid line).

show that they qualitatively accord with the FEG theory. By adjusting the electron density parameter $r_{s}$ we were able to achieve a good quantitative agreement between the electron lifetimes resulting from LMTO-GW calculations and from the FEG theory. Moreover, with such $r_{s}$ values agreement is also obtained for the hole lifetimes in $s, p$ states.

The lifetimes of holes in the top $d$ bands are much longer than the lifetimes calculated in the FEG theory, but they decrease rapidly with the decrease of energy. In the energy dependence of the hole lifetimes the two groups of quasiparticles in the states along the $X_{2}-\Gamma_{12}$ direction and the $W_{1}-X_{5}-L_{3}-\Gamma_{12}$ direction are clearly distinguished. We present for the first time the lifetime dispersion curves of $d$ holes. They show that phase space arguments work well in explaining the lifetime changes, although for some momenta the transition probabilities are also important.

The comparison with available experimental data on electron and hole lifetimes reveals, however, some disagreements, that can be related to effects not considered in the present model of quasiparticle damping; namely, excitonic processes, transport effects and electron-phonon interactions.

\section{ACKNOWLEDGMENTS}

We thank N. H. March for useful discussions and reading the manuscript. Partial support by the University of the Basque Country, the Basque Hezkuntza Saila, the Spanish Ministerio de Education y Cultura, the Max Planck Physics Award for International Cooperation and Iberdrola is gratefully acknowledged. F. A. acknowledges the financial support from the New Energy and Industrial Technology Development Organization (NEDO). 
${ }^{1}$ H. Petek and S. Ogawa, Prog. Surf. Sci. 56, 239 (1997).

${ }^{2}$ S. Hüfner, Photoemission Spectroscopy (Springer, Berlin, 1996).

${ }^{3}$ J. Bokor, Science 246, 1130 (1989); R. Hight, Surf. Sci. Rep. 21, 275 (1995).

${ }^{4}$ C.A. Schmuttenmaier, M. Aeschlimann, H.E. Elsayed-Ali, R.J.D. Miller, D.A. Mantell, J. Cao, and Y. Gao, Phys. Rev. B 50, 8957 (1994).

${ }^{5}$ T. Hertel, E. Knoesel, M. Wolf, and G. Ertl, Phys. Rev. Lett. 76, 535 (1996).

${ }^{6}$ S. Ogawa, H. Nagano, and H. Petek, Phys. Rev. B 55, 10869 (1997).

${ }^{7}$ J. Cao, Y. Gao, R.J.D. Miller, H.E. Elsayed-Ali, and D.A. Mantell, Phys. Rev. B 56, 1099 (1997).

${ }^{8}$ E. Knoesel, A. Hotzel, and M. Wolf, Phys. Rev. B 57, 12812 (1998)

${ }^{9}$ A. Goldmann, R. Matzdorf, and F. Theilmann, Surf. Sci. 414, L932 (1998).

${ }^{10}$ E. Knoesel, A. Hotzel, T. Hertel, and M. Wolf, Surf. Sci. 368, 76 (1996).

${ }^{11}$ J. Cao, Y. Gao, H.E. Elsayed-Ali, R.J.D. Miller, and D.A. Mantell, Phys. Rev. B 58, 10948 (1998).

${ }^{12}$ M. Aeschlimann, M. Bauer, S. Pawlik, W. Weber, R. Burgermeister, D. Oberli, and H.C. Siegmann, Phys. Rev. Lett. 79, 5158 (1997).

${ }^{13}$ R. Knorren, K.H. Bennemann, R. Burgermeister, and M. Aeschlimann, Phys. Rev. B 61, 9427 (2000).

${ }^{14}$ W. Nessler, S. Ogawa, H. Nagano, H. Petek, J. Shimoyama, Y. Nakayama, and K. Kishio, Phys. Rev. Lett. 81, 4480 (1998).

${ }^{15}$ J.J. Quinn and R.A. Ferrell, Phys. Rev. 112, 812 (1958); J.J. Quinn, ibid. 126, 1453 (1962).

${ }^{16}$ R.H. Ritchie, Phys. Rev. 114, 644 (1959); J.J. Quinn, ibid. 126, 1453 (1962); J.C. Shelton, Surf. Sci. 44, 305 (1974).

${ }^{17}$ R.H. Ritchie and J.C. Ashley, J. Phys. Chem. Solids 26, 1689 (1963); L. Kleinmann, Phys. Rev. B 3, 2982 (1971); D.R. Penn, ibid. 13, 5248 (1976); D.R. Penn, ibid. 22, 2677 (1980).

${ }^{18}$ C.J. Tung, J.C. Ashley, and R.H. Ritchie, Surf. Sci. 81, 427 (1979); D.R. Penn, Phys. Rev. B 35, 482 (1987).

${ }^{19}$ J.J. Quinn, Appl. Phys. Lett. 2, 167 (1963); S.L. Adler, Phys. Rev. 130, 1654 (1963).

${ }^{20}$ I. Campillo, A. Rubio, and J.M. Pitarke, Phys. Rev. B 59, 12188 (1999).

${ }^{21}$ W.-D. Schöne, R. Keyling, M. Bandič, and W. Ekardt, Phys. Rev. B 60, 8616 (1999)

${ }^{22}$ I. Campillo, J.M. Pitarke, A. Rubio, E. Zarate, and P.M. Echenique, Phys. Rev. Lett. 83, 2230 (1999).
${ }^{23}$ R. Keyling, W.-D. Schöne, and W. Ekardt, Phys. Rev. B 61, 1670 (2000).

${ }^{24}$ M.A. Cazalilla, J.S. Dolado, A. Rubio, and P.M. Echenique, Phys. Rev. B 61, 8033 (2000).

${ }^{25}$ I. Campillo, A. Rubio, J.M. Pitarke, A. Goldmann, and P.M. Echenique, Phys. Rev. Lett. 85, 3241 (2000).

${ }^{26}$ I. Campillo, V.M. Silkin, J.M. Pitarke, E.V. Chulkov, A. Rubio, and P.M. Echenique, Phys. Rev. B 61, 13484 (2000).

${ }^{27}$ L. Hedin and S. Lundqvist, in Solid State Physics, edited by H. Ehrenreich, F. Seitz, and D. Turnbull (Academic Press, New York, 1969), Vol. 23.

${ }^{28}$ F. Aryasetiawan and O. Gunnarsson, Rep. Prog. Phys. 61, 237 (1998).

${ }^{29}$ A. L. Fetter and J. D. Walecka, Quantum Theory of ManyParticle Systems (McGraw-Hill, New York, 1971).

${ }^{30}$ B.J. Lundqvist, Phys. Status Solidi 32, 273 (1969).

${ }^{31}$ F. Aryasetiawan and O. Gunnarsson, Phys. Rev. B 49, 7219 (1994).

${ }^{32}$ F. Aryasetiawan and O. Gunnarsson, Phys. Rev. B 49, 16214 (1994).

${ }^{33}$ F. Aryasetiawan, Phys. Rev. B 46, 13051 (1992).

${ }^{34}$ T. Miyake and F. Aryasetiawan, Phys. Rev. B 61, 7172 (2000).

${ }^{35}$ O.K. Andersen, Phys. Rev. B 12, 3060 (1975).

${ }^{36}$ O. K. Andersen, O. Jepsen, and M. Sob, in Electronic Band Structure and its Applications, Vol. 283 of the Lecture Notes in Physics, edited by M. Yussouff (Springer, Heidelberg, 1987).

${ }^{37}$ D. A. Papaconstantopoulos, Handbook on the Band Structures of Elemental Solids (Plenum Press, New York, 1986).

${ }^{38}$ J.A. Knapp, F.J. Himpsel, and D.E. Eastman, Phys. Rev. B 19, 4952 (1979).

${ }^{39}$ A. G. Eguiluz, J. M. Sullivan, and W. Ku, Int. Jou. Quantum Chem. (to be published 2001).

${ }^{40}$ E. D. Palik, Handbook of Optical Constants of Solids (Academic Press, New York, 1985), Vol. 2.

${ }^{41}$ M. Wolf and M. Aeschlimann, Phys. B1. 54, 145 (1998).

${ }^{42}$ W. Ekardt, W.-D. Schöne, and R. Keyling, Appl. Phys. A: Mater. Sci. Process. 71, 529 (2000).

${ }^{43}$ P.M. Echenique, J.M. Pitarke, E.V. Chulkov, and A. Rubio, Chem. Phys. 251, 1 (2000).

${ }^{44}$ H. Petek, H. Nagano, and S. Ogawa, Phys. Rev. Lett. 83, 832 (1999).

${ }^{45}$ R. Matzdorf, A. Gerlach, F. Theilmann, G. Meister, and A. Goldmann, Appl. Phys. B: Lasers Opt. 68, 393 (1999).

${ }^{46}$ B. Holms and U. von Barth, Phys. Rev. B 57, 2108 (1988). 